\title{
Facteurs de croissance et macrosomies : le temps des surprises
}

Dans le numéro de mars $1996\left(n^{\circ} 3\right.$, vol. 12) de médecine/sciences, plusieurs articles furent consacrés aux facteurs de croissance, et l'éditorial qui les présentait nous annonçait «le temps des surprises». Ce temps arrive, en effet. Non pas, comme on pouvait l'imaginer, par de nouvelles découvertes en biologie cellulaire, mais tout uniment grâce à l'étude d'une macrosomie humaine: le syndrome de Golabi-Rosen.

Parmi les macrosomies, seul jusqu'à présent, le syndrome de BeckwithWiedemann (BWS) avait retenu l'attention. Sa localisation en 11q15.5 (m/s $n^{\circ} 3$, vol 7, p. 292), une région soumise à l'empreinte parentale, et la présence du gène IGF2 (insulin growth factor) dans cette même région donnait l'envie d'y voir plus qu'une coïncidence. Du reste, une séduisante étude sur souris transgénique $\left(\mathrm{m} / \mathrm{s} n^{\circ} 10\right.$, vol. 11 , p. 1483) avait non seulement permis de comprendre le mécanisme de régulation de l'empreinte parentale inversée de $H 19$ et de $I g f 2$, mais aussi d'obtenir un modèle animal du BWS, les souris $i g f 2^{+/+}$, chez lesquelles les deux allèles $i g f 2$ s'expriment, ayant une taille $30 \%$ supérieure à la normale. Le syndrome de GolabiRosen, ou syndrome de SimpsonGolabi-Behmel (SGBS), encore appelé bulldog syndrome par les AngloSaxons, en raison de la dysmorphie faciale qui le caractérise (visage grossier et large, prognathisme) partage avec le BWS certains points communs: macrosomie, malformations, risque tumoral augmenté. Il est même parfois confondu avec lui [1]. Pourtant, un certain nombre de caractères distinctifs font la différence: l'excès de croissance, débutant en période prénatale et perceptible au troisième trimestre de la grossesse, se poursuit bien après la première décennie de la vie dans le SGBS, si bien que les adultes atteignent souvent 190 à $200 \mathrm{~cm}$. Alors que des tumeurs variées peuvent survenir dans le BWS, on n'observe dans le SGBS que deux types de tumeurs embryonnaires, la tumeur de Wilms et le neuroblastome. Parmi les malformations, les anomalies osseuses vertébrales (anomalie de Klippel Feil*), costales et l'hexadactylie sont surtout l'apanage du SGBS. Le quotient intellectuel est normal. Enfin, cette maladie ne touche que les garçons, et chaque fois que la transmission a pu être étudiée dans des familles, la liaison à l'X fut vérifiée, d'où son inscription dans OMIM (mendelian inheritance in man: http: //www.ncbi.nlm.nih.gov/omim/) parmi les maladies liées à l'X sous le $\mathrm{n}^{\circ} 312870$.

Comme il advient souvent dans les maladies liées à l'X, l'existence de deux femmes atteintes d'un SGBS et porteuses d'une translocation $\mathrm{X}$; autosome facilitait grandement la tâche [2]. En clonant les points de cassure sur l'X (tous deux situés en Xq25-26), on avait toutes chances de trouver le gène de cette maladie qui confine au gigantisme. Et, puisqu'il est porté par l'X, on imaginait volontiers un rôle régulateur à distance de l'empreinte de l'IGF. Il n'en est rien. Le groupe, à dominante italienne, qui vient $d$ 'isoler ce gène l'a identifié : il code pour une glypicane.

Comme vous ne l'avez certainement pas oublié, les facteurs de croissance sont reçus à la surface des cellules grâce à des co-récepteurs, les protéoglycanes à sulfate d'héparane
(HSPG) [3]. Celles qui sont associées à la membrane plasmatique (HSPGp) peuvent avoir un corps protéique transmembranaire, ce sont les syndécanes, ou être associées à la membrane par un groupement glycosyl phosphatidyl inositol (GPI), ce sont les glypicanes.

Après avoir obtenu un YAC contenant un des points de cassure, un gène fut isolé et séquencé, qui comporte $94 \%$ d'analogie avec OCI-5, codant pour une glypicane isolée dans l'intestin de rat [4]. La famille des glypicanes comportant déjà GPC1, la première glypicane trouvée chez l'homme, et GPC2, une cérébroglypicane [5], le gène fut baptisé $G P C 3$. Il est le premier à être associé à une maladie humaine. Contenant au moins huit exons, il s'étend sur plus de $500 \mathrm{~kb}$. Sa responsabilité dans le SGBS semble engagée puisqu'il est lésé dans tous les cas étudiés: dans les deux translocations ayant permis l'isolement du gène, l'un des points de cassure se situe dans le premier exon, et l'autre est dans le septième intron (ce qui devrait entraîner, pour la protéine, une perte d'environ 50 acides aminés). Dans trois autres familles où ségrège le SGBS, des délétions du gène furent retrouvées (de l'exon 2 , des exons 6,7 et 8 ). De plus, l'existence d'un complexe protéique GCP3-IGF2 est fort probable, puisqu'en utilisant une technique d'identification des protéines se liant à l'IGF et à l'aide d'anticorps antiGPC3, on trouve l'IGF2 marqué lié à

\footnotetext{
* Anomalie de Klippel Feil: fusions de vertèbres cervicales ayant pour conséquence un cou court avec limitation des mouvements de la tête.
} 
une protéine de taille analogue à la protéine potentielle.

Enfin, alors que GPC1 s'exprime dans de nombreux tissus et lignées cellulaires, et GPC2 en abondance dans le cerveau, l'expression de GPC3 n'est décelable que dans les tissus d'origine mésodermique.

Dès maintenant, il devient clair que GCP3 joue un rôle majeur dans la régulation de la croissance et qu'en son absence, l'activité d'IGF2 est augmentée. Mais quelles en sont les modalités précises? Comme l'expression de GPC3 et d'IFG2 est extrêmement faible dans les tissus adultes, il faudra recourir à des études sur la souris pour mieux comprendre les mécanismes régulateurs. On sait déjà qu'en cas de perte d' $I g f 2 r$, la taille de la souris est anormalement grande et que la suppression conjointe d'IGF2 normalise le phénotype [6]. En effet, l'IGF2 est un ligand du récepteur d'IGF1 qui relaie son action de facteur de croissance alors que le récepteur spécifique IGF2R est plutôt un modulateur négatif de l'action d'IGF2, entrant en compétition avec IGF1R pour la liaison du facteur de croissance. De la même manière, le glypicane 3 pourrait distraire, lui aussi, IGF2 de sa liaison au récepteur capable de transmettre un signal prolifératif (figure 1). Il faut à présent vérifier que la perte de GPC3 a pour conséquence ce même phénotype "grande taille» et que la suppression d'IGF2 normalise aussi ce phénotype. Si tel est bien le cas, le BWS et le syndrome de Golabi-Rosen seraient tous

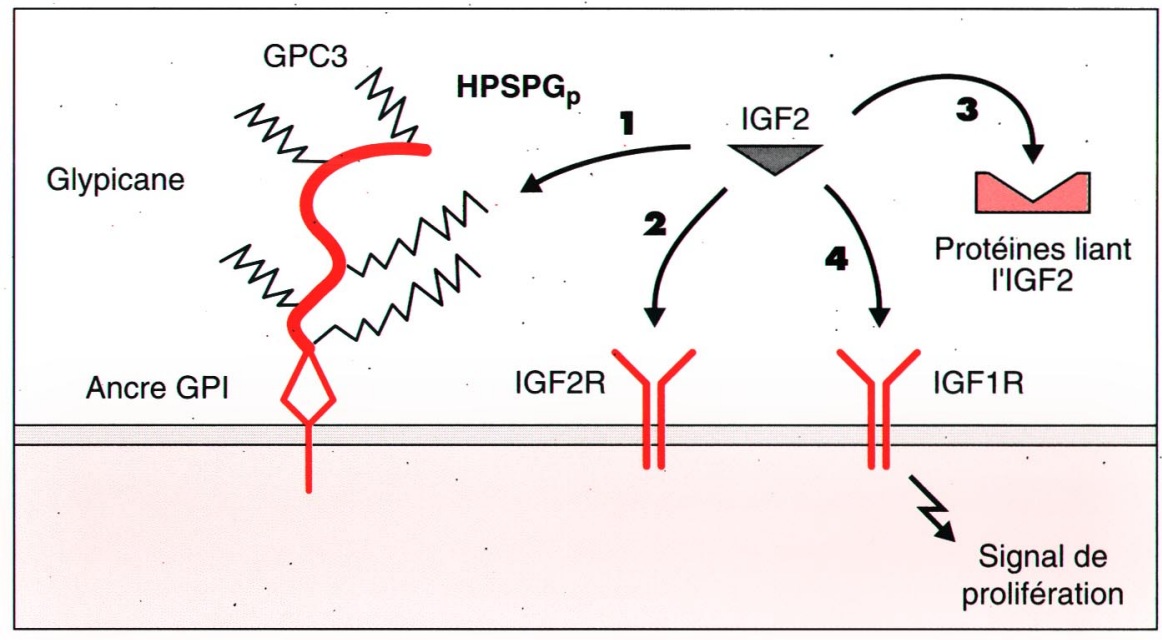

Figure 1. Rôles présomptifs de la glypicane en interaction avec le récepteur d'IGF2. GPC3 est ancré à la membrane par un groupement glycosyl phosphatidyl inositol (GPI). Le facteur de croissance IGF2 pourrait, soit être fixé par des protéines incapables de transmettre un signal prolifératif 11: le glypicane 3; 2: le récepteur (GF2R; 3: des protéines circulantes), soit se fixer au récepteur IGF1R, le seul à relayer un signal de prolifération. Ainsi, un signal prolifératif augmenté peut résulter d'une hyperproduction d'IGF2 ou d'un défaut de synthèse des protéines le distrayant de sa fixation sur IGF1R.

deux des maladies par excès de stimulation de la croissance par IGF2, hyperproduit dans le premier cas et insuffisamment stocké sous forme inactive dans le second.

S.G. cDNA corresponding to a developmentally regu-

1. Verloes A, Massard B, Dehalleux I, Langahendries JP, Koulischer L. Clinical overlap of Beck with Wiedemann, Perlman and Simpson GolabiBehmel syndromes: a diagnostic pitfall. Clin Genet 1995; 47: 257-62. lated transcript in rat intestine. Mol Cell Biol 1988; 8: 4243-9.

2. Punnet HH. Simpson Golabi-Behmel syndrocation. Am J Med Genet 1994; 50 : 391-3. nisme qui commence à être déchiffré. médecine/ sciences 1996 ; 12 : 303-12.

4. Filmus J, Church G,Buick RN. Isolation of a

5. David G. Integral membrane heparan sulfate proteoglycans. FASEB J 1993; 7 : 1023-30.

6. Baker J, Liu JP, Robertson EJ, Efstratiadis A. Role of insulin-like growth factors in embryonic and postnatal growth. Cell 1993; 75 : 73-82. me (SGBS) in a female with an autosome translo-

3. Blanquet PR. Les signaux des FGF: un méca-

\section{口曰 BRÈVES}

Une nouvelle fonction pour l'oncogène adénoviral E1A: l'inhibition de l'effet du TGF $\beta$. Le TGF $\beta$ se comporte comme un inhibiteur de la prolifération de la majorité des cellules épithéliales: il bloque le cycle cellulaire en phase G1, probablement par induction de l'activité de plusieurs inhibiteurs de protéines kinases Cdk actives sur le cycle cellulaire: $15^{\operatorname{Ink} 4 B}$ et $27^{\text {Kip } 1}$, ce dernier étant un inhibiteur du complexe entre la cycline $\mathrm{E}$ ou $\mathrm{A}$ et la kinase Cdk2 [1]. Le blocage de l'activité kinasique Cdk empêche la phosphorylation de la protéine $\mathrm{Rb}$, qui conserve ainsi son pouvoir d'inhibition du cycle cellulaire par liaison aux facteurs de transcription E2F. Mal et al., de Cleveland $(\mathrm{OH}$, USA) montrent, en travaillant sur des cellules épithéliales pulmonaires de vison, que la protéine E1A s'associe à l'inhibiteur $27^{\text {Kipl }}$ et l'inactive, bloquant ainsi l'action de TGF $\beta$ incapable d'inhiber l'activité du complexe cycline/Cdk2 [2]. Ces résultats montrent pour la première fois qu'un oncogène viral peut agir, notamment, par un blocage de l'action des inhibiteurs des complexes cyclines/Cdk.

[1. Darbon J, et al. médecine/sciences 1995; $11: 349-56$.]

[2. Mal A, et al. Nature 1996; 380, 262-5.] 
La maladie de von Hippel Lindau au Japon. La maladie de von Hippel Lindau (VHL) a connu un regain d'intérêt lors de la découverte du gène, localisé en 3p25-26, et elle a fait l'objet d'une synthèse dans nos colonnes [1]. Plus récemment, le mécanisme d'action du gène $V H L$ a été élucidé, gène suppresseur de tumeurs d'importance en raison de l'extrême variété des localisations tumorales: par une petite région colinéaire, la protéine VLH normale se lie à deux facteurs d'élongation transcriptionnelle, les élongines B et C $\left(m / s n^{\circ} 11\right.$, vol. $\left.11, p .1603\right)$. Pour la première fois, le groupe de recherche japonais sur la maladie de VHL publie ses travaux [2]. Dans la population japonaise, certaines mutations du gène $V H L$ sont identiques à celles déjà trouvées en Europe et aux États-Unis, mais les grandes délétions y sont moins fréquentes. Si les hémangiomes du système nerveux et de la rétine sont les manifestations les plus fréquentes, les cancers du rein et/ou les kystes du pancréas ne s'observent pas dans les familles avec phéochromocytome, et inversement. Or, la maladie de VHL avec phéochromocytome, que l'on classe actuellement comme maladie de VHL de type 2, n'existe que dans $9 \%$ des cas de la population japonaise contre $20 \%$ dans les populations européennes et, dans les familles avec phéochromocytomes, les altérations du gène $V L H$ sont toutes situées dans la partie carboxyterminale. La région située entre les codons 125 et 260 doit être déterminante pour ce type 2 de la maladie. La poursuite de l'étude des corrélations entre les manifestations cliniques et les différentes mutations permettra de mieux comprendre encore les mécanismes d'action du gène $V L H$.

[1. Richard S, et al. médecine/sciences 1995; $11: 43-51$.
[2. Clinical Research Group for VHL in Japan. Hum Mol Genet 1995; 4: 2233-7.]

[1 Élongation des transcrits et cancer: implication du facteur ELL dans les leucémies humaines. Le gène le plus fréquemment impliqué dans des réarrangements chromosomiques associés à des leucémies est $M L L$, également appelé ALL-1, HRX et Htrx, qui code pour un homologue humain du gène de drosophile trithorax $\left(m / s \quad n^{\circ} 11\right.$, vol. 9, p. 97). On lui connaît au moins sept partenaires de translocation dont l'un est le gène $E L L$. Une forme de leucémie myéloblastique aiguë est en effet associée à une translocation $\mathrm{t}(11 ; 19) \quad(\mathrm{q} 23$; p13.1), aboutissant à la formation d'un gène chimérique codant pour une protéine de fusion dont la partie aminoterminale est formée des 1300 premiers acides aminés de MLL et la partie carboxyterminale de l'essentiel de la région codée par le gène ELL. Shilatifard et al., du laboratoire de Joan et Ronald Conaway (Oklahoma City, OK, USA), sont parvenus à purifier partiellement une protéine de 80000 daltons se comportant comme un activateur de l'élongation des transcrits. Une séquence partielle de cette protéine devait la révéler identique à ELL. La protéine ELL recombinante est en effet dotée des mêmes propriétés d'activation de l'élongation des transcrits que le facteur purifié par l'équipe des Conaway [1]. ELL est le deuxième gène intervenant dans un phénomène de tumorigenèse chez l'homme à coder pour un facteur d'élongation. Rappelons que le premier, le produit du gène de susceptibilité à la maladie de von Hippel-Lindau (gène VHL) $(\mathrm{m} / \mathrm{s}$ $n^{\circ} 11$, vol. 11, p. 1603) était un partenaire inhibiteur du complexe d'élongation dénommé élongine.

[1. Shilatifard A, et al. Science 1996; 271 : 1873-6.] 